PROCEEDINGS OF THE

AMERICAN MATHEMATICAL SOCIETY

Volume 137, Number 12, December 2009, Pages 3969-3979

S 0002-9939(09)09965-1

Article electronically published on July 16, 2009

\title{
NORMAL HOPF SUBALGEBRAS OF SEMISIMPLE HOPF ALGEBRAS
}

\author{
SEBASTIAN BURCIU
}

(Communicated by Martin Lorenz)

\begin{abstract}
The notion of kernel of a representation of a semisimple Hopf algebra is introduced. Similar properties to those of the kernel of a group representation are proved in some special cases. In particular, every normal Hopf subalgebra of a semisimple Hopf algebra $H$ is the kernel of a representation of $H$. The maximal normal Hopf subalgebras of $H$ are described.
\end{abstract}

\section{INTRODUCTION}

In this paper the notion of kernel of a representation of a finite dimensional semisimple Hopf algebra is proposed. Similar properties to those of the kernel of a group representation are proved in some special cases.

Let $G$ be a finite group and $\mathrm{X}: G \rightarrow \operatorname{End}_{\mathbb{C}}(M)$ be a finite dimensional complex representation of $G$ which affords the character $\chi$. The kernel of the representation $M$ is defined as ker $\chi=\{g \in G \mid \chi(g)=\chi(1)\}$, and it is the set of all group elements $g \in G$ which act as the identity on $M$. (For example, see [2].) Every normal subgroup $N$ of $G$ is the kernel of a character, namely the character of the regular representation of $G / N$. If $\mathrm{Z}=\{g \in G|| \chi(g) \mid=\chi(1)\}$, then Z is called the center of the character $\chi$, and it is the set of group elements of $G$ which acts as a unit scalar on $M$. The properties of $\mathrm{Z}$ and ker $\chi$ are described in Lemma 2.27 2], which asserts that Z/ker $\chi$ is a cyclic subgroup of the center of $G / \operatorname{ker} \chi$.

If $M$ is a representation of a finite dimensional semisimple Hopf algebra $H$ and $\chi \in C(H)$ is its associated character, then ker $\chi \subset H$ is defined to be the set of all irreducible $H^{*}$-characters $d \in H$ such that $d$ acts as the scalar $\epsilon(d)$ on $M$. We prove that $\operatorname{ker} \chi=\left\{d \in \operatorname{Irr}\left(H^{*}\right) \mid \chi(d)=\epsilon(d) \chi(1)\right\}$. Similarly, the set of all irreducible $H^{*}$-characters $d \in H$ that act as a scalar of absolute value $\epsilon(d)$ on $M$ is characterized as $\mathrm{z}_{\chi}=\left\{d \in \operatorname{Irr}\left(H^{*}\right)|| \chi(d) \mid=\epsilon(d) \chi(1)\right\}$.

Section 1 presents the definitions and the main properties of the kernel of a character $\chi$ and its center $z_{\chi}$. It is shown that these sets of characters are closed under multiplication and under the duality operation " $*$ ". Thus they generate Hopf subalgebras of $H$, denoted by $H_{\chi}$ and $\mathrm{Z}_{\chi}$, respectively. We say that a Hopf

Received by the editors October 18, 2007, and, in revised form, March 9, 2009.

2000 Mathematics Subject Classification. Primary 16W30.

Key words and phrases. Hopf algebras, normal subalgebras, central characters.

This research was supported by grant CEx05-D11-11/04.10.05 from the Ministry of Education and Research, Romania.

(C)2009 American Mathematical Society Reverts to public domain 28 years from publication 
subalgebra $K$ of $H$ is the kernel of a representation if $K=H_{\chi}$ for a certain character $\chi$ of $H$.

Section[2]studies the relationship between normal Hopf subalgebras and the Hopf algebras generated by kernels. It is shown that any normal Hopf subalgebra is the kernel of a character which is central in $H^{*}$.

In Section 3 the converse of the above statement is proven. More precisely, it is shown that for a representation $M$ of $H$ affording a character $\chi$ which is central in $H^{*}$, the Hopf subalgebra $H_{\chi}$ is normal in $H$. This implies that a Hopf subalgebra is normal if and only if it is the kernel of a character which is central in the dual Hopf algebra. Under the same assumption on $\chi$ it is shown that the irreducible representations of $H / / H_{\chi}:=H / H H_{\chi}^{+}$are precisely the irreducible representations of $H$ which are constituents of some tensor power of $M$. Using a basis description given in [14] for the algebra generated by the characters which are central in $H^{*}$, we describe a finite collection of normal Hopf subalgebras of $H$ which are the maximal normal Hopf subalgebras of $H$ (under inclusion). Any other normal Hopf subalgebra is an intersection of some of these Hopf algebras. Two other results that hold for group representations are presented in this section.

In this paper we work over the base field $\mathbb{C}$. For a vector space $V,|V|$ denotes the dimension $\operatorname{dim}_{\mathbb{C}} V$. We use Sweedler's notation, $\Delta(x)=\sum x_{1} \otimes x_{2}$, for comultiplication. All other notation is the same as that used in [7.

\section{Properties of the Kernel}

Let $H$ be a finite dimensional semisimple Hopf algebra over $\mathbb{C}$. Then $H$ is also cosemisimple [5]. Denote by $\operatorname{Irr}(H)$ the set of irreducible characters of $H$ and by $C(H)$ the character ring of $H$. Then $C(H)$ is a semisimple subalgebra of $H^{*}$, [15] and $C(H)=\operatorname{Cocom}\left(H^{*}\right)$, the space of cocommutative elements of $H^{*}$. By duality, the character ring of $H^{*}$ is a semisimple subalgebra of $H$, and under this identification it follows that $C\left(H^{*}\right)=\operatorname{Cocom}(H)$. If $M$ is an $H$-module with character $\chi$, then $M^{*}$ is also an $H$-module with character $\chi^{*}=\chi \circ S$. This induces an involution "*" : $C(H) \rightarrow C(H)$ on $C(H)$.

Recall that the exponent of $H$ is the smallest positive number $m>0$ such that $h^{[m]}=\epsilon(h) 1$ for all $h \in H$. The generalized power $h^{[m]}$ is defined by $h^{[m]}=$ $\sum_{(h)} h_{1} h_{2} \ldots h_{m}$. The exponent of a finite dimensional semisimple Hopf algebra is always finite and divides the third power of the dimension of $H$ [1].

Remark 1.1. Let $W$ be a simple $H^{*}$-module. Then $W$ is a simple right $H$-comodule, and one can associate to it a simple subcoalgebra of $H$ denoted by $C_{W}$ [4. If $q=|W|$, then $\left|C_{W}\right|=q^{2}$ and $C_{W}$ is a matrix coalgebra. It has a basis $\left\{x_{i j}\right\}_{1 \leq i, j \leq q}$ such that $\Delta\left(x_{i j}\right)=\sum_{l=1}^{q} x_{i l} \otimes x_{l j}$ for all $1 \leq i, j \leq q$. Moreover, $W \cong \mathbb{C}<x_{1 i} \mid 1 \leq$ $i \leq q>$ as right $H$-comodules, where $\rho\left(x_{1 i}\right)=\Delta\left(x_{1 i}\right)=\sum_{l=1}^{q} x_{1 l} \otimes x_{l i}$ for all $1 \leq i \leq q$. The character of $W$ as a left $H^{*}$-module is $d \in C\left(H^{*}\right) \subset H$, and it is given by $d=\sum_{i=1}^{q} x_{i i}$. Then $\epsilon(d)=q$, and the simple subcoalgebra $C_{W}$ is also denoted by $C_{d}$.

Proposition 1.2. Let $H$ be a finite dimensional semisimple Hopf algebra and let $M$ be a representation of $H$ affording the character $\chi \in C(H)$. If $W$ is an irreducible representation of $H^{*}$ affording the character $d \in C\left(H^{*}\right)$, then the following hold:

(1) $|\chi(d)| \leq \chi(1) \epsilon(d)$. 
(2) Equality holds if and only if $d$ acts as $\alpha \epsilon(d) \operatorname{Id}_{M}$ on $M$ for some root of unity $\alpha \in \mathbb{C}$.

Proof. (1) $W$ is a right $H$-comodule, and one can define a map $\mathrm{T}$ which is similar to the one defined in Paragraph 3.1 of [3]:

$$
\begin{aligned}
\mathrm{T}: M \otimes W & \longrightarrow \\
m \otimes w & \longmapsto \sum w_{1} m \otimes w_{0} .
\end{aligned}
$$

It can be checked that $\mathrm{T}^{p}(m \otimes w)=\sum w_{1}^{[p]} m \otimes w_{0}$ for all $p \geq 0$. Thus, if $m=\exp (H)$, then $\mathrm{T}^{m}=\operatorname{Id}_{M \otimes W}$. Therefore $\mathrm{T}$ is a semisimple operator and all its eigenvalues are roots of unity. It follows that $\operatorname{tr}(\mathrm{T})$ is the sum of all these eigenvalues and consequently $|\operatorname{tr}(\mathrm{T})| \leq \operatorname{dim}_{\mathbb{C}}(M \otimes W)=\chi(1) \epsilon(d)$. It is easy to see that $\operatorname{tr}(\mathrm{T})=\chi(d)$. Indeed, using the above remark one can suppose that $W=\mathbb{C}<x_{1 i} \mid 1 \leq i \leq q>$ where $C_{W}=\mathbb{C}<x_{i j} \mid 1 \leq i, j \leq$ $q>$ is the coalgebra associated to $W$. Then the formula for $\mathrm{T}$ becomes $\mathrm{T}\left(m \otimes x_{1 i}\right)=\sum_{j=1}^{\epsilon(d)} x_{j i} m \otimes x_{1 j}$, which shows that $\operatorname{tr}(\mathrm{T})=\sum_{i=1}^{\epsilon(d)} \chi\left(x_{i i}\right)=$ $\chi(d)$.

(2) Equality holds if and only if $\mathrm{T}=\alpha \mathrm{Id}_{M \otimes W}$ for some root of unity $\alpha$. The above expression for $\mathrm{T}$ implies that in this case $x_{i j} m=\delta_{i, j} \alpha m$ for any $1 \leq i, j \leq \epsilon(d)$. Therefore $d m=\alpha \epsilon(d) m$ for any $m \in M$. The converse is immediate.

Let $M$ be a representation of $H$ which affords the character $\chi$. Define ker $\chi$ as the set of all irreducible characters $d \in \operatorname{Irr}\left(H^{*}\right)$ which act as the scalar $\epsilon(d)$ on $M$. The previous proposition implies that ker $\chi=\left\{d \in \operatorname{Irr}\left(H^{*}\right) \mid \chi(d)=\epsilon(d) \chi(1)\right\}$. Similarly let $\mathrm{z}_{\chi}$ be the set of all irreducible characters $d \in \operatorname{Irr}\left(H^{*}\right)$ which act as a scalar $\alpha \epsilon(d)$ on $M$, where $\alpha$ is a root of unity. Then from the same proposition it follows that $\mathrm{z}_{\chi}=\left\{d \in \operatorname{Irr}\left(H^{*}\right)|| \chi(d) \mid=\epsilon(d) \chi(1)\right\}$. Clearly ker $\chi \subset \mathrm{z}_{\chi}$.

Remark 1.3. (1) The proof of Proposition 1.2 implies that for a representation $M$ of $H$ affording a character $\chi \in C(H)$ and an irreducible character $d \in$ $\operatorname{Irr}\left(H^{*}\right)$ the following assertions are equivalent:

1) $d \in$ ker $\chi$.

2) $\chi(d)=\epsilon(d) \chi(1)$.

3) $\chi\left(x_{i j}\right)=\delta_{i j} \chi(1)$ for all $i, j$.

4) $d m=\epsilon(d) m$ for all $m \in M$.

5) $x_{i j} m=\delta_{i j} m$ for all $i, j$ and all $m \in M$.

(2) Similarly one has the following equivalences:

1) $d \in \mathrm{z}_{\chi}$.

2) $|\chi(d)|=\epsilon(d) \chi(1)$.

3) There is a root of unity $\alpha \in \mathbb{C}$ such that $\chi\left(x_{i j}\right)=\alpha \delta_{i j} \chi(1)$ for all $i, j$.

4) There is a root of unity $\alpha \in \mathbb{C}$ such that $d m=\alpha \epsilon(d) m$ for all $m \in M$.

5) There is a root of unity $\alpha \in \mathbb{C}$ such that $x_{i j} m=\alpha \delta_{i j} m$ for all $i, j$ and all $m \in M$.

(3) Let $\operatorname{Irr}(H)=\left\{\chi_{0}, \cdots, \chi_{s}\right\}$ be the set of all irreducible $H$-characters and let $M$ be a representation of $H$ which affords the character $\chi$. If $\chi=\sum_{i=0}^{s} m_{i} \chi_{i}$ where $m_{i} \in \mathbb{Z}_{\geq 0}$, then ker $\chi=\bigcap_{m_{i} \neq 0}$ ker $\chi_{i} \subset \mathrm{z}_{\chi} \subset$ $\bigcap_{m_{i} \neq 0} \mathrm{z}_{\chi_{i}}$. 
A subset $X \subset \operatorname{Irr}\left(H^{*}\right)$ is closed under multiplication if for every $\chi, \mu \in X$ in the decomposition of $\chi \mu=\sum_{\gamma \in \operatorname{Irr}\left(H^{*}\right)} m_{\gamma} \gamma$ one has $\gamma \in X$ if $m_{\gamma} \neq 0$. A subset $X \subset \operatorname{Irr}\left(H^{*}\right)$ is closed under "*" if $x^{*} \in X$ for all $x \in X$.

Proposition 1.4. Let $H$ be a finite dimensional semisimple Hopf algebra and $M$ a representation of $H$ affording the character $\chi \in C(H)$. Then the subsets ker $\chi$ and $\mathrm{z}_{\chi}$ of $\operatorname{Irr}\left(H^{*}\right)$ are closed under multiplication and under "*".

Proof. Proposition 1.2 implies that $\chi(d)=\epsilon(d) \chi(1)$ if and only if $d$ acts as $\epsilon(d) \operatorname{Id}_{M}$ on $M$. Therefore if $d \in \operatorname{ker} \chi$, then $d^{*}=S(d) \in \operatorname{ker} \chi$ since $\chi\left(d^{*}\right)=\overline{\chi(d)}[10$. Let $d, d^{\prime} \in$ ker $\chi$. Then $d d^{\prime}$ acts as $\epsilon\left(d d^{\prime}\right) \operatorname{Id}_{M}$ on $M$ since $d$ acts as $\epsilon(d) \operatorname{Id}_{M}$ and $d^{\prime}$ acts as $\epsilon\left(d^{\prime}\right) \operatorname{Id}_{M}$ on $M$. Write $d d^{\prime}=\sum_{i=1}^{q} m_{i} d_{i}$ where $d_{i}$ are irreducible characters of $H^{*}$ and $m_{i} \neq 0$ for all $1 \leq i \leq q$. Then $\chi\left(d d^{\prime}\right)=\sum_{i=1}^{q} m_{i} \chi\left(d_{i}\right)$ and

$$
\chi(1) \epsilon\left(d d^{\prime}\right)=\left|\chi\left(d d^{\prime}\right)\right| \leq \sum_{i=1}^{q} m_{i}\left|\chi\left(d_{i}\right)\right| \leq \chi(1) \sum_{i=1}^{q} m_{i} \epsilon\left(d_{i}\right)=\chi(1) \epsilon\left(d d^{\prime}\right) .
$$

It follows from Proposition 1.2 that $\chi\left(d_{i}\right)=\chi(1) \epsilon\left(d_{i}\right)$ and therefore $d_{i} \in$ ker $\chi$ for all $1 \leq i \leq q$. The proof for $\mathrm{z}_{\chi}$ is similar.

Remark 1.5. (1) For later use let us note that ker $\chi \subset$ ker $\chi^{n}$ for all $n \geq$ 0 . Indeed if $d \in \operatorname{Irr}\left(H^{*}\right)$ is an element of ker $\chi$, then one has a simple subcoalgebra $C_{d}$ associated to $d$ and $d=\sum_{i=1}^{\epsilon(d)} x_{i i}$. Item (11) of Remark 1.3 implies that $\chi\left(x_{i j}\right)=\chi(1) \delta_{i j}$. Thus

$$
\chi^{n}(d)=\sum_{i=1}^{\epsilon(d)} \sum_{i_{1}, \cdots, i_{n-1}=1}^{\epsilon(d)} \chi\left(x_{i i_{1}}\right) \chi\left(x_{i_{1} i_{2}}\right) \cdots \chi\left(x_{i_{n-1} i}\right)=\chi(1)^{n} \epsilon(d) .
$$

Similarly it can be shown that $\mathrm{z}_{\chi} \subset \mathrm{z}_{\chi^{n}}$ for all $n \geq 0$.

(2) If $X \subset \operatorname{Irr}\left(H^{*}\right)$ is closed under multiplication and under "* ", then it generates a Hopf subalgebra of $H$ denoted by $H_{X}[9]$. One has $H_{X}=\bigoplus_{d \in X} C_{d}$. Using this, since the sets ker $\chi$ and $z_{\chi}$ are closed under multiplication and under " * ", they generate Hopf subalgebras of $H$ denoted by $H_{\chi}$ and $\mathrm{Z}_{\chi}$, respectively.

(3) The proof of Proposition 1.2 implies that $\chi \downarrow_{H_{\chi}}=\chi(1) \epsilon_{H_{\chi}}$, where $\chi \downarrow_{H_{\chi}}$ is the restriction of $\chi$ to the subalgebra $H_{\chi}$ and $\epsilon_{H_{\chi}}$ is the character of the trivial module over the Hopf algebra $H_{\chi}$.

(4) Suppose that $M$ and $N$ are two $H$-modules affording the characters $\chi$ and $\mu$. If $M$ is a submodule of $N$, then it can easily be seen that $\operatorname{ker} \mu \subset \operatorname{ker} \chi$ and consequently that $H_{\mu} \subset H_{\chi}$.

\section{Normal Hopf Subalgebras}

As before let $H$ be a finite dimensional semisimple Hopf algebra over $\mathbb{C}$. We use the notation $\Lambda_{H} \in H$ for the idempotent integral of $H\left(\epsilon\left(\Lambda_{H}\right)=1\right)$ and $t_{H} \in H^{*}$ for the idempotent integral of $H^{*}\left(t_{H}(1)=1\right)$. From Proposition 4.1 of 4 it follows that the regular character of $H$ is given by the formula

$$
|H| t_{H}=\sum_{\chi \in \operatorname{Irr}(H)} \chi(1) \chi .
$$


The dual formula is

$$
|H| \Lambda_{H}=\sum_{d \in \operatorname{Irr}\left(H^{*}\right)} \epsilon(d) d
$$

One also has $t_{H}\left(\Lambda_{H}\right)=\frac{1}{|H|}[5]$.

If $K$ is a Hopf subalgebra of $H$, then $K$ is a semisimple and cosemisimple Hopf algebra [7. A Hopf subalgebra $K$ of $H$ is called normal if $h_{1} x S\left(h_{2}\right) \in K$ and $S\left(h_{1}\right) x h_{2} \in K$ for all $x \in K$ and $h \in H$. If $H$ is a semisimple Hopf algebra as above, then $S^{2}=$ Id (see [5) and $K$ is normal in $H$ if and only if $h_{1} x S\left(h_{2}\right) \in K$ for all $x \in K$ and $h \in H$. If $K^{+}=\operatorname{Ker}(\epsilon) \cap K$, then $K$ is a normal Hopf subalgebra of $H$ if and only if $H K^{+}=K^{+} H$. In this situation $H / / K:=H / H K^{+}$is a quotient Hopf algebra of $H$ via the canonical map $\pi: H \rightarrow H / / K$ (see Lemma 3.4.2 of [7). In our setting, $K$ is normal in $H$ if and only if $\Lambda_{K}$ is central in $H$ (see Lemma 1 on page 1932 of [6]).

Remark 2.3. Suppose that $K$ is a normal Hopf subalgebra of $H$ and let $L=H / / K$ be the quotient Hopf algebra of $H$ via $\pi: H \rightarrow L$. Then $\pi^{*}: L^{*} \rightarrow H^{*}$ is an injective Hopf algebra map. It follows that $\pi^{*}\left(L^{*}\right)$ is normal in $H^{*}$ and it is easy to see that $\left(H^{*} / / L^{*}\right)^{*} \cong K$. The representations of $L=H / / K$ are those representations $M$ of $H$ such that each $x \in K$ acts as $\epsilon(x) \operatorname{Id}_{M}$ on $M$. If $\chi$ is the character of $M$ as an $L$-module, then $\pi^{*}(\chi) \in C(H)$ is the character of $M$ as an $H$-module and with the above notation, $H_{\pi^{*}(\chi)} \supset K$.

Notation: Let $\mu$ be any irreducible character of $H$ and let $\xi_{\mu} \in \mathrm{Z}(H)$ be the central primitive idempotent associated to it. Then $\nu\left(\xi_{\mu}\right)=\delta_{\mu, \nu} \mu(1)$ for any other irreducible character $\nu$ of $H$ and $\left\{\xi_{\mu}\right\}_{\mu \in \operatorname{Irr}(H)}$ is the complete set of central orthogonal idempotents of $H$. Dually, since $H^{*}$ is semisimple to any irreducible character $d \in C\left(H^{*}\right)$, one has an associated central primitive idempotent $\xi_{d} \in \mathrm{Z}\left(H^{*}\right)$. As before one can view $d$ as being in $H^{* *}=H$, and the above relation becomes $\xi_{d}\left(d^{\prime}\right)=\delta_{d, d^{\prime}} \epsilon(d)$ for any other irreducible character $d^{\prime} \in C\left(H^{*}\right)$. Also $\left\{\xi_{d}\right\}_{d \in \operatorname{Irr}\left(H^{*}\right)}$ is the complete set of central orthogonal idempotents of $H^{*}$.

We say that a Hopf subalgebra $K$ of $H$ is the kernel of a character if $K=H_{\chi}$ for some character $\chi \in C(H)$. The following is the main result of this section.

Theorem 2.4. Let $H$ be a finite dimensional semisimple Hopf algebra. Any normal Hopf subalgebra $K$ of $H$ is the kernel of a character which is central in $H^{*}$. More precisely, with the above notation one has

$$
K=H_{|L| \pi^{*}\left(t_{L}\right)} .
$$

Proof. Let $L=H / / K$. Then $L$ is a semisimple and cosemisimple Hopf algebra [7. The above remark shows that the representations of $L$ are exactly those representations $M$ of $H$ such that $H_{\chi_{M}} \supset K$ where $\chi_{M}$ is the $H$-character of $M$. Let $\pi: H \rightarrow L$ be the natural projection and let $\pi^{*}: L^{*} \rightarrow H^{*}$ be its dual map. Then $\pi^{*}$ is an injective Hopf algebra map and $L^{*}$ can be identified with a Hopf subalgebra of $H^{*}$. Therefore, if $t_{L} \in L^{*}$ is the idempotent integral of $L$, then $|L| t_{L}$ is the regular character of $L$ and $H_{|L| \pi^{*}\left(t_{L}\right)} \supset K$. Since $\pi^{*}\left(L^{*}\right)$ is a normal Hopf subalgebra of $H^{*}$ it follows that $\pi^{*}\left(t_{L}\right)$ is a central element of $H^{*}$. We have to show that $H_{|L| \pi^{*}\left(t_{L}\right)}=K$ and the proof will be complete. 
With the above notation, since $\pi^{*}\left(t_{L}\right)$ is a central idempotent of $H^{*}$, one can write it as a sum of central primitive orthogonal idempotents:

$$
\pi^{*}\left(t_{L}\right)=\sum_{d \in X} \xi_{d}
$$

where $X$ is a subset of $\operatorname{Irr}\left(H^{*}\right)$. It follows that for any $d \in \operatorname{Irr}\left(H^{*}\right)$ one has that $\pi^{*}\left(t_{L}\right)(d)=\epsilon(d)$ if $d \in X$ and $\pi^{*}\left(t_{L}\right)(d)=0$ otherwise, which shows that $X=$ ker $|L| \pi^{*}\left(t_{L}\right)$. Since $H_{|L| \pi^{*}\left(t_{L}\right)} \supset K$, one has $\pi^{*}\left(t_{L}\right)(d)=\epsilon(d)$ for all $d \in \operatorname{Irr}\left(K^{*}\right)$ and thus $\operatorname{Irr}\left(K^{*}\right) \subset X$. Let $\Lambda_{H} \in H$ and $\Lambda_{L} \in L$ be the idempotent integrals of $H$ and $L$. Since $\pi$ is a surjective Hopf algebra map, one has $\pi\left(\Lambda_{H}\right)=\Lambda_{L}$. Then

$$
\pi^{*}\left(t_{L}\right)\left(\Lambda_{H}\right)=t_{L}\left(\pi\left(\Lambda_{H}\right)\right)=t_{L}\left(\Lambda_{L}\right)=\frac{1}{|L|} .
$$

On the other hand, since $\Lambda_{H}=\frac{1}{|H|} \sum_{d \in \operatorname{Irr}\left(H^{*}\right)} \epsilon(d) d$ it follows that

$$
\pi^{*}\left(t_{L}\right)\left(\Lambda_{H}\right)=\frac{1}{|H|} \sum_{d \in X} \epsilon(d)^{2},
$$

which implies that $\sum_{d \in X} \epsilon(d)^{2}=\frac{|H|}{|L|}=|K|$. Since $\sum_{d \in \operatorname{Irr}\left(K^{*}\right)} \epsilon(d)^{2}=|K|$ and $\operatorname{Irr}\left(K^{*}\right) \subset X$, we conclude that $\operatorname{Irr}\left(K^{*}\right)=X$ and $H_{|L| \pi^{*}\left(t_{L}\right)}=K$.

Let $\mathbb{C}$ be the trivial $K$-module via the augmentation map $\epsilon_{K}$. Denote by $\epsilon \uparrow_{K}^{H}:=$ $\epsilon_{K} \uparrow_{K}^{H}$ the character of the induced module $H \otimes_{K} \mathbb{C}$.

Corollary 2.5. Let $K$ be a Hopf subalgebra of $H$. Then $K$ is normal in $H$ if and only if $H_{\epsilon \uparrow \uparrow_{K}^{H}}=K$.

Proof. Suppose $K$ is a normal Hopf subalgebra of $H$. With the notation from the above theorem, since $\epsilon \uparrow_{K}^{H}=|L| \pi^{*}\left(t_{L}\right)$ and $H_{|L| \pi^{*}\left(t_{L}\right)}=K$, it follows that $H_{\epsilon \uparrow_{K}^{H}}=K$. Conversely, suppose that $H_{\epsilon \uparrow_{K}^{H}}=K$. Then by using the third item of Remark 1.5]it follows that $\epsilon \uparrow_{K}^{H} \downarrow_{K}^{H}=\frac{|H|}{|K|} \epsilon_{K}$. Using Frobenius reciprocity this implies that for any irreducible character $\chi$ of $H$ the value of $m\left(\chi \downarrow_{K}, \epsilon_{K}\right)=m\left(\chi, \epsilon \uparrow_{K}^{H}\right)$ is either $\chi(1)$ if $\chi$ is a constituent of $\epsilon \uparrow_{K}^{H}$ or 0 otherwise. But if $\Lambda_{K}$ is the idempotent integral of $K$, then $m\left(\chi \downarrow_{K}, \epsilon_{K}\right)=\chi\left(\Lambda_{K}\right)$. Thus $\chi\left(\Lambda_{K}\right)$ is either zero or $\chi(1)$ for any irreducible character $\chi$ of $H$. This implies that $\Lambda_{K}$ is a central idempotent of $H$ and therefore that $K$ is a normal Hopf subalgebra of $H$ by [6] (see also Proposition 1.7.2 of [ $[$ ] ).

\section{Central characters}

Let $H$ be a finite dimensional semisimple Hopf algebra over $\mathbb{C}$. Consider the central subalgebra of $H$ defined by $\hat{\mathrm{Z}}(H)=\mathrm{Z}(H) \cap C\left(H^{*}\right)$. It is the algebra of $H^{*}$-characters which are central in $H$. Let $\hat{\mathrm{Z}}\left(H^{*}\right):=\mathrm{Z}\left(H^{*}\right) \cap C(H)$ be the dual object, namely the subalgebra of $H$-characters which are central in $H^{*}$.

Let $\phi: H^{*} \rightarrow H$ be given by $f \mapsto f \rightarrow \Lambda_{H}$ where $f \rightarrow \Lambda_{H}=f\left(S\left(\Lambda_{H_{1}}\right)\right) \Lambda_{H_{2}}$. Then $\phi$ is an isomorphism of vector spaces [7].

Remark 3.1. With the notation from the previous section, it can be checked that $\phi\left(\xi_{d}\right)=\frac{\epsilon(d)}{|H|} d^{*}$ and $\phi^{-1}\left(\xi_{\chi}\right)=\chi(1) \chi$ for all $d \in \operatorname{Irr}\left(H^{*}\right)$ and $\chi \in \operatorname{Irr}(H)$ (see for example [7]). 
We use the following description of $\hat{\mathrm{Z}}\left(H^{*}\right)$ and $\hat{\mathrm{Z}}(H)$ as given in [14. Since $\phi(C(H))=\mathrm{Z}(H)$ and $\phi\left(\mathrm{Z}\left(H^{*}\right)\right)=C\left(H^{*}\right)$, it follows that the restriction

$$
\left.\phi\right|_{\hat{\mathbf{Z}}\left(H^{*}\right)}: \hat{\mathrm{Z}}\left(H^{*}\right) \rightarrow \hat{\mathrm{Z}}(H)
$$

is an isomorphism of vector spaces.

Since $\hat{\mathrm{Z}}\left(H^{*}\right)$ is a commutative semisimple algebra it has a vector space basis given by its primitive idempotents. Since $\hat{\mathrm{Z}}\left(H^{*}\right)$ is a subalgebra of $\mathrm{Z}\left(H^{*}\right)$ each primitive idempotent of $\hat{\mathrm{Z}}\left(H^{*}\right)$ is a sum of primitive idempotents of $\mathrm{Z}\left(H^{*}\right)$. But the primitive idempotents of $\mathrm{Z}\left(H^{*}\right)$ are of the form $\xi_{d}$ where $d \in \operatorname{Irr}\left(H^{*}\right)$. Thus, there is a partition $\left\{\mathcal{Y}_{j}\right\}_{j \in J}$ of the set of irreducible characters of $H^{*}$ such that the elements $\left(e_{j}\right)_{j \in J}$ given by

$$
e_{j}=\sum_{d \in \mathcal{Y}_{j}} \xi_{d}
$$

form a basis for $\hat{\mathrm{Z}}\left(H^{*}\right)$. Note that $e_{j}(d)=\epsilon(d)$ if $d \in \mathcal{Y}_{j}$ and $e_{j}(d)=0$ if $d \notin \mathcal{Y}_{j}$. Since $\phi\left(\hat{\mathrm{Z}}\left(H^{*}\right)\right)=\hat{\mathrm{Z}}(H)$ it follows that $\widehat{e}_{j}:=|H| \phi\left(e_{j}\right)$ is a basis for $\hat{\mathrm{Z}}(H)$. Using the first formula from Remark 3.1 one has

$$
\widehat{e}_{j}=\sum_{d \in \mathcal{Y}_{j}} \epsilon(d) d^{*}
$$

Remark 3.2. (1) By duality, the set of irreducible characters of $H$ can be partitioned into a finite collection of subsets $\left\{\mathcal{X}_{i}\right\}_{i \in I}$ such that the elements $\left(f_{i}\right)_{i \in I}$ given by

$$
f_{i}=\sum_{\chi \in \mathcal{X}_{i}} \chi(1) \chi
$$

form a $\mathbb{C}$-basis for $\hat{\mathrm{Z}}\left(H^{*}\right)$. Then the elements $\phi\left(f_{i}\right)=\sum_{\chi \in \mathcal{X}_{i}} \xi_{\chi}$ are the central orthogonal primitive idempotents of $\hat{\mathrm{Z}}(H)$ and therefore form a basis for this space. Clearly $|I|=|J|$.

(2) Let $M$ be a representation of a semisimple Hopf algebra $H$. Consider the set $\mathcal{C}$ of all simple representations of $H$ which are direct summands in some tensor power $M^{\otimes n}$. Then $\mathcal{C}$ is closed under tensor products and under "* " and thus generates a Hopf algebra $L$ which is a quotient of $H$ (see [12] or 13]). Note that if $\mathcal{C} \subset \operatorname{Irr}(H)$ is closed under multiplication and under "**", then from the dual version of item (2) of Remark 1.5 it follows that $\mathcal{C}$ generates a Hopf subalgebra $H_{\mathcal{C}}^{*}$ of $H^{*}$. It follows that $L=\left(H_{\mathcal{C}}^{*}\right)^{*}$ (see also Proposition 3.11 of [11]). If $M$ has character $\chi \in H^{*}$, then the character $\pi^{*}\left(t_{L}\right) \in C(H)$ can be expressed as a polynomial in $\chi$ with rational coefficients (see Corollary 19 of [10]).

Proposition 3.3. Suppose $\chi$ is a character of $H$ which is central in $H^{*}$. Then $H_{\chi}$ is a normal Hopf subalgebra of $H$ and the simple representations of $H / / H_{\chi}$ are the simple constituents of all the powers of $\chi$.

Proof. Since $\chi \in \hat{\mathrm{Z}}\left(H^{*}\right)$, with the above notation one has $\chi=\sum_{j \in J} \alpha_{j} e_{j}$, where $\alpha_{j} \in \mathbb{C}$. It follows that $\chi(d)=\alpha_{j} \epsilon(d)$ if $d \in \mathcal{Y}_{j}$. Therefore if $d \in \mathcal{Y}_{j}$, then $d \in \operatorname{ker} \chi$ if and only if $\alpha_{j}=\chi(1)$. This implies that ker $\chi$ is the union of all the sets $\mathcal{Y}_{j}$ such that $\alpha_{j}=\chi(1)$. Using formula (2.2) the integral $\left|H_{\chi}\right| \Lambda_{H_{\chi}}$ can be written as

$$
\left|H_{\chi}\right| \Lambda_{H_{\chi}}=\sum_{d \in \operatorname{ker} \chi} \epsilon(d) d=\sum_{\left\{j \mid \alpha_{j}=\chi(1)\right\}} \sum_{d \in \mathcal{Y}_{j}} \epsilon(d) d
$$


and therefore

$$
\left|H_{\chi}\right| \Lambda_{H_{\chi}}=\left|H_{\chi}\right| S\left(\Lambda_{H_{\chi}}\right)=\sum_{\left\{j \mid \alpha_{j}=\chi(1)\right\}} \sum_{d \in \mathcal{Y}_{j}} \epsilon(d) d^{*}=\sum_{\left\{j \mid \alpha_{j}=\chi(1)\right\}} \widehat{e}_{j} .
$$

Then $\Lambda_{H_{\chi}}$ is central in $H$ since each $\widehat{e}_{j}$ is central in $H$. As above this implies that $H_{\chi}$ is normal in $H$.

Let $V$ be an $H$-module with character $\chi$ and let $I=\bigcap_{m>0} \operatorname{Ann}\left(V^{\otimes m}\right)$. If $L$ is the quotient Hopf algebra of $H$ generated by the constituents of all the powers of $\chi$, then from [13] or [12] one has that $L=H / I$. Note that $I \supset H H_{|L| \pi^{*}\left(t_{L}\right)}^{+}$. Using item (2) of Remark 3.2 one obtains that $\pi^{*}\left(t_{L}\right)$ is a polynomial in $\chi$ with rational coefficients. Since $\chi$ is central in $H^{*}$ it follows that $\pi^{*}\left(t_{L}\right)$ is a central element of $H^{*}$ and thus that $L^{*}$ is a normal Hopf subalgebra of $H^{*}$. Using (2.3) (for $L^{*} \hookrightarrow H^{*}$ ), it follows that $H / /\left(H^{*} / / L^{*}\right)^{*}=L$. Then if $K=\left(H^{*} / / L^{*}\right)^{*}$, one has $H / / K=L$. Theorem 2.4 implies that $H_{|L| \pi^{*}\left(t_{L}\right)}=K$; thus $H / / H_{|L| \pi^{*}\left(t_{L}\right)}=H / / K=L$. But $L=H / I$, and since $I \supset H H_{|L| \pi^{*}\left(t_{L}\right)}^{+}$it follows that $H H_{|L| \pi^{*}\left(t_{L}\right)}^{+}=I$. It is easy to see that $H_{\chi}^{+} \subset I$ since the elements of $H_{\chi}$ act as $\epsilon$ on each tensor power of $V$ (see item (1) of Remark 1.5).

On the other hand $|L| t_{L}$ is the regular character of $L$. Then ker $\chi \supset$ ker $|L| \pi^{*}\left(t_{L}\right)$ since $\chi$ is a constituent of $|L| \pi^{*}\left(t_{L}\right)$. Thus $I \supset H H_{\chi}^{+} \supset H H_{|L| \pi^{*}\left(t_{L}\right)}^{+}$. Since $H H_{|L| \pi^{*}\left(t_{L}\right)}^{+}=I$ it follows that $H H_{\chi}^{+}=I$ and thus $H / / H_{\chi}=L$.

Theorem 2.4 and the previous proposition imply the following corollary:

Corollary 3.4. A Hopf subalgebra of $H$ is normal if and only if it is the kernel of a character $\chi$ which is central in $H^{*}$.

Let $H_{i}:=H_{f_{i}}$; see Remark 3.2 for the definition of $f_{i}$. From Proposition 3.3 it follows that $H_{i}$ is a normal Hopf subalgebra of $H$. If $K$ is any other normal Hopf subalgebra of $H$, then Theorem 2.4 implies that $K=H_{\chi}$ for some central character $\chi$. Following [14, one has $\chi=\sum_{i \in I^{\prime}} m_{i} f_{i}$ for some rational positive numbers $m_{i}$ and some subset $I^{\prime} \subset I$. Then ker $\chi=\bigcap_{i \in I^{\prime}}$ ker $f_{i}$, which implies that $H_{\chi}=\bigcap_{i \in I^{\prime}} H_{i}$. Thus any normal Hopf subalgebra is an intersection of some of these Hopf algebras $H_{i}$.

Remark 3.5. If $K$ and $L$ are normal Hopf subalgebras of $H$, then it is easy to see that $K L=L K$ is a normal Hopf subalgebra of $H$ that contains both $K$ and $L$.

Let $L$ be any Hopf subalgebra of $H$. We define core $(L)$ to be the biggest Hopf subalgebra of $L$ which is normal in $H$. Based on Remark 3.5 clearly core $(L)$ exists and is unique. If $A$ is a Hopf subalgebra of $H$, then there is an isomorphism of $H$-modules $H / H A^{+} \cong H \otimes_{A} \mathbb{C}$ given by $\bar{h} \mapsto h \otimes_{A} 1$. Thus if $A \subset B \subset H$ are Hopf subalgebras of $H$, then $\epsilon \uparrow_{B}^{H}$ is a constituent of $\epsilon \uparrow_{A}^{H}$ since there is a surjective $H$-module map $H / H A^{+} \rightarrow H / H B^{+}$.

Remark 3.6. Suppose $\chi$ and $\mu$ are the characters of two representations $M$ and $N$ of $H$ such that $\mu$ is central in $H^{*}$ and $\chi$ is an irreducible character which is a constituent of $\mu$. From item (11) of Remark 3.2 it follows that $\chi \in \mathcal{X}_{i_{0}}$ for some $i_{0} \in I$. Since $\mu$ is central in $H^{*}$ it follows that $\mu$ is a linear combination with nonnegative rational coefficients of the elements $f_{i}$. Since $\chi$ is a constituent of $\mu$ it follows that $f_{i_{0}}$ is also a constituent of an integral multiple of $\mu$. Thus ker $\mu \subset \operatorname{ker} f_{i_{0}}$. 
Theorem 3.7. If $\chi$ is an irreducible character of $H$ such that $\chi \in \mathcal{X}_{i}$ for some $i \in I$, then $\operatorname{core}\left(H_{\chi}\right)=H_{f_{i}}$.

Proof. Let $K=\operatorname{core}\left(H_{\chi}\right)$. Since $\chi$ is a constituent of $f_{i}$, by item (4) of Remark 1.5 one has that $H_{f_{i}} \subset H_{\chi}$. The normality of $H_{f_{i}}$ implies that $H_{f_{i}} \subset K$. By the proof of Corollary $2.5 \mu:=\epsilon \uparrow_{K}^{H}$ is the $H$-character of $H / / K=H / K^{+} H$ and is central in $H^{*}$. Since $\chi \downarrow_{H_{\chi}}=\chi(1) \epsilon_{H_{\chi}}$ and $K \subset H_{\chi}$ it follows that $\chi \downarrow_{K}=\chi(1) \epsilon_{K}$. By Frobenius reciprocity one has that $\chi$ is a constituent of the character $\mu$. Using Remark 3.6 we then have ker $f_{i} \supset \operatorname{ker} \mu$ and $H_{f_{i}} \supset H_{\mu}=K$.

Remark 3.8. Item (3) of Remark 1.5 implies that $\chi$ is a constituent of $\epsilon \uparrow_{H_{\chi}}^{H}$ and therefore that $H_{\chi} \supseteq H_{\epsilon \uparrow H_{\chi}}$. Let $H_{1}=H_{\chi}$ and

$$
H_{s+1}=H_{\epsilon \uparrow \uparrow_{s}^{H}} \text { for } s \geq 1 \text {. }
$$

The above argument implies that $H_{s} \supseteq H_{s+1}$. Since $H$ is finite dimensional we conclude that there is $l \geq 1$ such that $H_{l}=H_{l+1}=\cdots=H_{l+n}=\cdots$. Corollary 2.5 gives that $H_{l}$ is a normal Hopf subalgebra of $H$. We claim that core $\left(H_{\chi}\right)=H_{l}$. Indeed, for any normal Hopf subalgebra $K$ of $H$ with $K \subset H_{\chi} \subset H$ we have that $\epsilon \uparrow_{H_{\chi}}^{H}$ is a constituent of $\epsilon \uparrow_{K}^{H}$, and then using Corollary 2.5 it follows that $K=H_{\epsilon \uparrow T_{K}^{H}}^{x} \subseteq H_{\epsilon \uparrow_{H_{\chi}}^{H}}=H_{2}$. Inductively, it can be shown that $K \subset H_{s}$ for any $s \geq 1$, which implies that $\operatorname{core}\left(H_{\chi}\right)=H_{l}$.

Proposition 3.9. Let $H$ be a semisimple Hopf algebra. Then

$$
\bigcap_{\chi \in \operatorname{Irr}(H)} \mathrm{z}_{\chi}=\bar{G}(H)
$$

where $\bar{G}(H)$ is the set of all central grouplike elements of $H$.

Proof. Any central grouplike element $g$ of $H$ acts as a scalar on each simple $H$ module. Since $g^{\exp (H)}=1$ it follows that this scalar is a root of unity and then

$$
\bar{G}(H) \subset \bigcap_{\chi \in \operatorname{Irr}(H)} \mathrm{z}_{\chi} .
$$

Let $d \in \bigcap_{\chi \in \operatorname{Irr}(H)} \mathrm{z}_{\chi}$. If $C_{d}$ is the simple subcoalgebra of $H$ associated to $d$ (see Remark 1.11), then $d=\sum_{i=1}^{\epsilon(d)} x_{i i}$. Item (2) of Remark 1.3 implies that $x_{i j}$ acts as $\delta_{i, j} \alpha_{\chi} \operatorname{Id}_{M_{\chi}}$ on $M_{\chi}$ where $\alpha_{\chi}$ is a root of unity. For $i \neq j$, it follows that $x_{i j}$ acts as zero on each irreducible representation of $H$. Therefore $x_{i j}=0$ for all $i \neq j$ and $d$ is a grouplike element of $H$. Since $d$ acts as a scalar on each irreducible representation of $H$ we have $d \in \mathrm{Z}(H)$ and therefore $d \in \bar{G}(H)$.

The next theorem is the generalization of the fact that $\mathrm{Z} /$ ker $\chi$ is a cyclic subgroup of $G /$ ker $\chi$ for any character of the finite group $G$.

Theorem 3.10. Let $M$ be a representation of $H$ such that its character $\chi$ is central in $H^{*}$. Then $\mathrm{Z}_{\chi}$ is a normal Hopf subalgebra of $H$ and $\mathrm{Z}_{\chi} / / H_{\chi}$ is the group algebra of a cyclic subgroup of $\mathbb{C} \bar{G}\left(H / / H_{\chi}\right)$. 
Proof. Since $\chi \in \hat{\mathrm{Z}}\left(H^{*}\right)$ one can write $\chi=\sum_{j \in J} \alpha_{j} e_{j}$ with $\alpha_{j} \in \mathbb{C}$. A similar argument to the one in Proposition 3.3 shows that

$$
\left|\mathrm{Z}_{\chi}\right| \Lambda_{\mathrm{z}_{\chi}}=\left|\mathrm{Z}_{\chi}\right| S\left(\Lambda_{\mathrm{z}_{\chi}}\right)=\sum_{\left\{j|| \alpha_{j} \mid=\chi(1)\right\}} \sum_{d \in \mathcal{Y}_{j}} \epsilon(d) d^{*}=\sum_{\left\{j|| \alpha_{j} \mid=\chi(1)\right\}} \widehat{e}_{j} .
$$

Therefore $\Lambda_{\mathrm{z}_{\chi}}$ is central in $H$ and $\mathrm{Z}_{\chi}$ is a normal Hopf subalgebra of $H$. Let $\pi: H \rightarrow H / / H_{\chi}$ be the canonical projection. Since $H$ is a free $\mathrm{Z}_{\chi}$-module there is also an injective Hopf algebra map $i: \mathrm{Z}_{\chi} / / H_{\chi} \rightarrow H / / H_{\chi}$ such that $i(\bar{z})=\pi(z)$ for all $z \in \mathrm{Z}_{\chi}$. Proposition 3.3 implies that the irreducible representations of $H / / H_{\chi}$ are precisely the irreducible constituents of tensor powers of $\chi$. From item (11) of Remark 1.5] it follows that $\mathrm{Z}_{\chi} \subset \mathrm{Z}_{\chi^{l}}$ for any nonnegative integer $l$. Let $d \in \mathrm{z}_{\chi}$ and let $C_{d}=\left\langle x_{i j}\right\rangle$ be the coalgebra associated to $d$ as in Remark 1.1. Item (2) of Remark 1.3 implies that for $i \neq j$ the element $x_{i j}$ acts as zero on any tensor power of $\chi$ and therefore its image under $\pi$ is zero. Since $\pi$ is a coalgebra map, one has

$$
\Delta\left(\pi\left(x_{i i}\right)\right)=\sum_{j=1}^{\epsilon(d)} \pi\left(x_{i j}\right) \otimes \pi\left(x_{j i}\right)=\pi\left(x_{i i}\right) \otimes \pi\left(x_{i i}\right) .
$$

Thus $\pi\left(x_{i i}\right)$ is a grouplike element of $H / / H_{\chi}$. Since $\pi\left(x_{i i}\right)$ acts as a scalar on each irreducible representation of $H / / H_{\chi}$ it follows that $\pi\left(x_{i i}\right)$ is a central grouplike element of $H / / H_{\chi}$. This proves that the image under $i$ of $\mathrm{Z}_{\chi} / / H_{\chi}$ is inside $\mathbb{C} \bar{G}\left(H / / H_{\chi}\right)$. The grouplike elements that act as a scalar on the representation $M$ of $H / / H_{\chi}$ form a cyclic group by Theorem 5.4 of [3] and the proof is finished.

Remark 3.11. If $\chi \in \hat{\mathrm{Z}}\left(H^{*}\right)$ is an irreducible character of $H$, then Proposition 3.3 together with Theorem 5.4 of $\left[3\right.$ implies that $\bar{G}\left(H / / H_{\chi}\right)$ is a cyclic group of order equal to the index of the character $\chi$.

\section{ACKNOWLEDGMENTS}

The author thanks the referee for valuable comments and suggestions made on the first version of this paper.

\section{REFERENCES}

1. P. Etingof and S. Gelaki, On the exponent of finite-dimensional Hopf algebras, Math. Res. Lett. 6 (1999), no. 2, 131-140. MR.1689203 (2000f:16045)

2. I. M. Isaacs, Character theory of finite groups, vol. 69, Pure and Applied Mathematics, Academic Press, New York-London, 1976. MR 0460423 (57:417)

3. Y. Kashina, Y. Sommerhäuser, and Y. Zhu, On higher Frobenius-Schur indicators, Mem. Amer. Math. Soc. 181 (2006), no. 855. MR.2213320 (2007k:16071)

4. R. G. Larson, Characters of Hopf algebras, J. Algebra 17 (1971), 352-368. MR0283054 $(44: 287)$

5. R. G. Larson and D. E. Radford, Finite-dimensional cosemisimple Hopf algebras in characteristic 0 are semisimple, J. Algebra 117 (1988), no. 2, 267-289. MR957441 (89k:16016)

6. A. Masuoka, Semisimple Hopf algebras of dimension 2p, Comm. Algebra 23 (1995), no. 5, 1931-1940. MR1323710 (96e:16050)

7. S. Montgomery, Hopf algebras and their actions on rings, vol. 82, CBMS Regional Conference Series in Mathematics, Amer. Math. Soc, Providence, RI, 1993. MR,1243637 (94i:16019)

8. S. Natale, Semisolvability of semisimple Hopf algebras of low dimension, Mem. Amer. Math. Soc. 186 (2007), no. 874. MR2294999 (2008b:16066)

9. W. D. Nichols and M. B. Richmond, The Grothendieck group of a Hopf algebra, J. Pure Appl. Algebra 106 (1996), no. 3, 297-306. MR1375826(97a:16075) 
10. , The Grothendieck algebra of a Hopf algebra. I, Comm. Algebra 26 (1998), no. 4, 1081-1095. MR:1612188 (99m:16064)

11. D. Nikshych, $K_{0}$-rings and twisting of finite-dimensional semisimple Hopf algebras, Comm. Algebra 26 (1998), no. 1, 321-342. MR.1600702 (99d:16045a)

12. D. S. Passman and D. Quinn, Burnside's theorem for Hopf algebras, Proc. Amer. Math. Soc. 123 (1995), no. 2, 327-333. MR1215204 (95c:16050)

13. M. A. Rieffel, Burnside's theorem for representations of Hopf algebras, J. Algebra 6 (1967), 123-130. MR0210794 (35:1680)

14. S. Zhu, On finite-dimensional semisimple Hopf algebras, Comm. Algebra 21 (1993), no. 11, 3871-3885. MR1238131 (95d:16057)

15. Y. Zhu, Hopf algebras of prime dimension, Internat. Math. Res. Notices 1 (1994), 53-59. MR:1255253 (94j:16072)

Institute of Mathematics "Simion Stollow" of the Romanian Academy, P.O. Box 1-764, RO-014700, Bucharest, Romania

E-mail address: smburciu@syr.edu 